DOI: https://doi.org/10.11144/Javeriana.upsy16-4.agcc

\title{
Ansiedad, gestión conductual del conflicto y estrategias de afrontamiento en profesionales de la orientación laboral ${ }^{*}$
}

\section{Anxiety, Behavioral Conflict Management and Coping Strategies in Occupational Guidance Professionals}

Yolanda NAVARRo-AbAL ${ }^{\mathrm{a}}$

Universidad de Huelva, España

ORCID: http://orcid.org/0000-0002-0438-844X

José Antonio Climent-Rodríguez

Universidad de Huelva, España

Ma José López-López

Universidad de Huelva, España

a Autor de correspondencia. Correo electrónico: yolanda.navarro@dpsi.uhu.es

Para citar este artículo: Navarro-Abal, Y., ClimentRodríguez, J., \& López-López, M.J. (2017). Ansiedad, gestión conductual del conflicto y estrategias de afrontamiento en profesionales de la orientación laboral. Universitas Psychologica, 16(4), 1-8. https://do i.org/10.11144/Javeriana.upsy16-4.agcc

\section{RESUMEN}

En el desempeño de sus funciones de atención a personas sin empleo, el orientador laboral se encuentra sometido a diversos y frecuentes estresores generadores de ansiedad; uno de estos estresores son los conflictos. Utilizando una muestra compuesta por 90 orientadores laborales del Servicio de Empleo de Andalucía (España), este estudio pretende poner de manifiesto en qué medida la ansiedad manifestada se encuentra relacionada con los estilos de gestión conductual del conflicto adoptado, y este con las estrategias de afrontamiento al estrés. Los resultados muestran elevados niveles de ansiedad (estado y rasgo) en este colectivo, así como asociaciones importantes entre las estrategias de afrontamiento al estrés en general y los estilos de gestión de conflictos, lo que indica que ambos se encuentran conceptualmente relacionados.

Palabras clave

estrés; ansiedad; orientación laboral; gestión del conflicto.

\section{ABSTRACT}

The employment consultant is normally exposed, when he performs his/ her function attending to people unemployed, to different and common stressors, one of these stressors are the conflicts. Using a sample of 90 guidance workers of the Andalusian Employment Service (Spain), this study aims to show to what extent the exposed anxiety is related to behavioral conflict management style, and this last with strategies of coping with stress. Results show high levels of anxiety, as well as significant relations between strategies of coping with stress and behavioral styles of management of conflicts, pointing out that they are related conceptually. Keywords

stress; anxiety; job guidance; conflict management. 
En los últimos años, en España ha tenido lugar el desarrollo de un nuevo perfil profesional que surge con el objetivo de apoyar a las personas desempleadas o subempleadas con el objetivo de facilitar tanto su inserción socio-laboral como el desarrollo de su carrera profesional (De Pablo, 1995; Fernández-Garrido, 2010); se trata de la figura del orientador laboral u orientador profesional para el empleo. Su trabajo supone una combinación de actividades de asesoramiento y consejo a las personas demandantes de empleo (Cuntigh, Pérez \& Personnaz, 2006), junto con labores de mejora del afrontamiento y modificación de posibles actitudes negativas (Di Fabio \& Bernaud, 2008). Como profesión nueva en España, el ámbito de la orientación laboral tiene un escaso desarrollo a nivel de construcción profesional, así como también son escasos los trabajos dedicados a los riesgos laborales y amenazas a la salud en dicho colectivo. No obstante, estudios franceses desarrollados bajo auspicios sindicales (Pole Emploi, 2009) reflejan la incidencia de la ansiedad y el estrés en los trabajadores de la orientación laboral, al vincular dicho estrés a factores de riesgo como la intensificación del trabajo (expresada por el aumento de demandantes de empleo a atender, la realización de dicha actividad en un periodo de tiempo cada vez menor e incluso, con reducción del personal disponible), así como la ansiedad generada por la interacción frecuente con personas a su vez altamente ansiosas y estresadas, en algunos caso poco colaboradoras e incluso, violentas. Es importante tener en cuenta que muchos profesionales concentran su actividad en colectivos con especiales dificultades (desempleados de larga duración, marginación social, enfermedad mental, etc.), y al igual que ocurre en otros profesionales de la intervención social, las principales herramientas de quienes trabajan en orientación laboral son sus competencias de comunicación e interacción, de las que dependen, así como del adecuado afrontamiento que lleven a cabo para controlar o reducir los niveles de estrés (Maslach, Schaufeli, \& Leiter, 2001; Schaufeli, 1998). En este sentido, es de sobra conocida la relevancia de las estrategias de afrontamiento como amortiguadoras del potencial efecto del estrés sobre la salud (Basler, Kaluza, \& LledóBoyer, 2003; Dewe, 1989; Lazarus, 2000). En los últimos años, en España la violencia ocupacional, también denominada violencia laboral de origen externo, (Organización Internacional del Trabajo, 2003) ha aumentado de manera significativa en la administración pública. Dentro del sector público, se observa una situación especialmente preocupante por la frecuencia y gravedad de los episodios de violencia hacia los trabajadores en los centros sanitarios y en las oficinas y centros de empleo, en los que realizan su trabajo la plantilla de orientadores laborales. Todo ello ha llevado al propio Gobierno de la Nación, además de a muchos otros gobiernos autonómicos, a establecer medidas legislativas de prevención y manejo de estas situaciones de manera específica, especialmente en las oficinas de empleo, en donde se establece que estas situaciones un rol relevante como componente mediador entre las circunstancias estresantes y la salud un rol relevante como componente mediador entre las circunstancias estresantes y la salud, que coinciden con la grave crisis económica y de empleo a la que se ha visto sometido el país y que, especialmente, ha castigado a la parte de la población con mayor vulnerabilidad socioeconómica y laboral (Secretaría de Estado de Administraciones Públicas, 2015).

Por otra parte, teniendo en cuenta que un gran número de actividades y deberes de la orientación laboral se concentra en la intervención con personas desempleadas inmersas, con frecuencia, en situaciones complejas a nivel personal, familiar, económico y social, un conjunto de competencias claves de la figura del orientador laboral radica en la adecuada negociación entre el profesional y la persona usuaria para tomar decisiones acerca de su inserción laboral; es decir, para el orientador laboral, como integrante del equipo de profesionales de la Oficina de Empleo que centra su labor en la atención directa a personas usuarias, el conflicto resulta prácticamente consustancial a la propia dinámica organizacional, donde se 
establece su manejo y gestión como parte de un proceso que emana de la rutina laboral diaria (Munduate \& Medina, 2006), y es uno de los principales desencadenantes de ansiedad y estrés en cualquier profesional de la intervención social (Lázaro, 2004). En este sentido, diferentes estudios (De Dreu, Van Dierendonck, \& Dijskstra, 2004; Van Woerkom \& Van Engen, 2009) han corroborado el hecho de que la gestión inadecuada de los conflictos tiene consecuencias negativas para la salud de los trabajadores y la productividad de la organización, pues los conflictos negativos se convierten en estresores de larga duración, que generan emociones negativas como la ira, tristeza o ansiedad (Spector, Chen, \& O'Connell, 2000), y dan lugar a problemas físicos y emocionales.

Por tanto, teniendo en cuenta los potenciales factores de riesgo inherentes a la orientación laboral expuestos hasta ahora, y sobre todo la carencia de trabajos donde se aborde el estudio de variables de funcionamiento personal en este colectivo, planteamos esta investigación a nivel descriptivo y exploratorio. Los objetivos son evaluar los niveles de ansiedad que presentan los orientadores laborales, así como la relación entre estos y los estilos conductuales utilizados en la gestión de conflictos. Al tratarse estos de una situación estresante generadora de ansiedad, nos planteamos también estudiar la relación entre las estrategias utilizadas en el afrontamiento de situaciones estresantes en general y la gestión o afrontamiento de los conflictos en particular.

\section{Método}

\section{Participantes}

La muestra está compuesta por un total de 90 orientadores laborales que prestan sus servicios en distintos centros de empleo de la provincia de Huelva. Del total de participantes, 23 son hombres y 67 mujeres $(25.5 \%$ y $74.5 \%$, respectivamente); la edad oscila entre 25 y 59 años $(M=43.14 ; D T=10.4)$.

\section{Instrumentos}

Todos los participantes cumplimentan los siguientes instrumentos:

Cuestionario de Ansiedad Estado-Rasgo (STAI; Spielberger, Gorsuch, \& Lushore, 1970). Se utiliza la adaptación al castellano de Seisdedos (1988), que consta de 40 ítems en formato de respuesta tipo Lickert de 3 puntos, distribuidos en dos subescalas, Ansiedadestado (STAI-E) y Ansiedad-Rasgo (STAIR). Este instrumento presenta unas adecuadas propiedades psicométricas, con una elevada consistencia interna (entre 0.9 y 0.93 en Ansiedad-Estado y entre 0.84 y 0.87 en Ansiedad-Rasgo).

Inventario Rahim Organizational Conflict Inventory-II (ROCI-II; Rahim, 1983). Se utiliza la adaptación española de Munduate, Ganaza y Alcaide (1993), la cual consta de 28 ítems (con un formato de respuesta tipo Lickert de cinco puntos), distribuidos en cinco escalas de estilos de gestión conductual de conflictos: Integración, Compromiso, Dominación, Evitación y Servilismo. Presenta una adecuada consistencia interna, en la que el alfa de Cronbach oscilando en las diferentes escalas entre 0.62 y 0.77 .

Inventario de Estrategias de Afrontamiento al Estrés (CSI; Tobin, Holroyd, Reynold, \& Kigal, 1989). Utilizamos la adaptación de Cano, Rodríguez y García (2006), formada por 72 ítems (que se responden en una escala tipo Lickert de 5 puntos), agrupados en ocho escalas primarias: Resolución de Problemas, Autocrítica, Expresión Emocional, Pensamiento Desiderativo, Apoyo Social, Restructuración Cognitiva, Evitación de Problemas y Retirada Social. La consistencia interna de las escalas es elevada, y el alfa de Cronbach oscila entre 0.72 y 0.94 .

\section{Procedimiento}

En primer lugar, se contacta con los responsables técnicos de la Orientación Laboral del Servicio Andaluz de Empleo dependiente de la Junta de Andalucía, a quienes se les informa de los objetivos de la investigación. Una vez concedida 
la pertinente autorización por parte de los responsables del servicio, se procede a remitir por correo electrónico a los 108 orientadores laborales de Huelva y provincia, la solicitud de colaboración en el estudio junto con el correspondiente consentimiento informado y los diferentes instrumentos de evaluación; de los 108 orientadores, 90 remiten los cuestionarios correctamente cumplimentados.

\section{Resultados}

En primer lugar, en la Tabla 1 aparecen las puntuaciones obtenidas por los orientadores laborales en los tres cuestionarios. En relación al STAI, podemos observar que la puntuación media obtenida por los orientadores en Ansiedad-Rasgo es de 27.45 (DT = 4.87), mientras que la puntuación media en AnsiedadEstado es de $45.3(D T=4.74)$.

\section{TABLA 1}

Descriptivos del STAI, ROCI-II, SCI y de sus respectivas escalas

\begin{tabular}{lll}
\hline & Media & DT \\
\hline STAI & 27.45 & 4.87 \\
Rasgo & 45.3 & 4.74 \\
Estado & & \\
ROCI II & & \\
Integración & 2.09 & 0.71 \\
Evitación & 3.82 & 0.67 \\
Dominación & 2.94 & 0.91 \\
Servilismo & 2.73 & 0.58 \\
Compromiso & 1.94 & 0.62 \\
SCI & & \\
Resolución de & 18.4 & 3.39 \\
problemas & & \\
Reestructuración & 16.41 & 4.82 \\
Cognitiva & & \\
Expresión de & 17.5 & 3.74 \\
emociones & & \\
Apoyo social & 16.5 & 2.95 \\
Evitación de & 18.52 & 4.23 \\
problemas & & \\
Pensamiento & 19.8 & 3.77 \\
desiderativo & & \\
Autocritica & 12.53 & 2.98 \\
Retirada social & 14.38 & 4.42 \\
\hline
\end{tabular}

Fuente: elaboración propia.

Con respecto a los estilos de gestión conductual de los conflictos, así como a las estrategias de afrontamiento del estrés, en la misma Tabla 1 observamos que el estilo más utilizado por los orientadores es Evitación ( $\mathrm{M}=$ 3.82; DT $=0.67)$, mientras que la estrategia de afrontamiento que obtiene la puntuación más elevada es Pensamiento desiderativo $(\mathrm{M}=19.8$; $\mathrm{DT}=3.77)$ seguida de Evitación de problemas $(\mathrm{M}$ $=18.52 ; \mathrm{DT}=4.23$ ).

A continuación, en la Tabla 2 encontramos la correspondiente matriz de correlaciones obtenida entre el STAI y el ROCI-II. Tal y como podemos observar, encontramos que tanto la AnsiedadRasgo como la Ansiedad-Estado correlacionan positivamente con los siguientes estilos de gestión conductual de conflictos: Evitación, Dominación y Servilismo.

TABLA 2

Matriz de correlaciones entre las escalas del STAI y ROCI-II

\begin{tabular}{|c|c|c|c|c|c|c|c|}
\hline & 1 & 2 & 3 & 4 & 5 & 6 & 7 \\
\hline \multirow{2}{*}{\multicolumn{8}{|c|}{$\begin{array}{l}1 . \\
\text { Ansiedad-Rasgo }\end{array}$}} \\
\hline & & & & & & & \\
\hline 2. & 0.03 & & & & & & \\
\hline Ansiedad-Estado & & & & & & & \\
\hline 3. Evitación & $0.14^{*}$ & $0.18^{*}$ & & & & & \\
\hline 4. Compromiso & 0 & 0 & 0.08 & & & & \\
\hline 5. Dominación & $0.25^{* *}$ & $0.27^{* *}$ & 0.09 & 0.04 & & & \\
\hline 6. Servilismo & $0.18^{*}$ & $0.19^{*}$ & 0.01 & 0.01 & 0.02 & & \\
\hline 7. Integración & 0 & 0 & 0 & 0 & 0 & 0 & \\
\hline
\end{tabular}

Por último, en la Tabla 3 aparece la relación obtenida entre los estilos de gestión conductual de conflictos y las estrategias de afrontamiento. Tal y como se pone de manifiesto, la utilización del estilo Evitación correlaciona positivamente con las estrategias de afrontamiento de Evitación de problemas $(\mathrm{r}=0.15 ; \mathrm{p}<0.05)$, Pensamiento desiderativo $(\mathrm{r}=0.16 ; \mathrm{p}<0.05)$, Autocrítica $(\mathrm{r}=0.19 ; \mathrm{p}<0.05)$ y Retirada social $(\mathrm{r}=$ $0.16 ; \mathrm{p}<0.05)$. De la misma forma, se halla relación negativa con Reestructuración cognitiva $(\mathrm{r}=-0.14 ; \mathrm{p}<0.05)$. Por su parte, el estilo de Compromiso, correlaciona en sentido positivo con las estrategias de afrontamiento de Resolución de problemas $(\mathrm{r}=0.27 ; \mathrm{p}<0.01)$, Reestructuración cognitiva $(\mathrm{r}=0.19 ; \mathrm{p}<0.01)$, Expresión de emociones $(\mathrm{r}=0.21 ; \mathrm{p}<0.01)$, Apoyo social $(\mathrm{r}=0.22 ; \mathrm{p}<0.01)$ y Autocrítica $(\mathrm{r}=0.15$; 
$\mathrm{p}<0.01)$, mientras que con Retirada Social, lo hace en sentido negativo $(\mathrm{r}=-0.19 ; \mathrm{p}<0.05)$. Por su parte, el estilo de Dominación, se asocia positivamente con las estrategias de Expresión de emociones $(\mathrm{r}=0.24 ; \mathrm{p}<0.01)$ y Pensamiento desiderativo $(\mathrm{r}=0.18 ; \mathrm{p}<0.05)$, y en sentido negativo con la utilización del Apoyo social $(\mathrm{r}=$ -0.14; $\mathrm{p}<0.05$ ). En cuanto a Servilismo, muestra correlaciones positivas con las estrategias de Evitación de problemas $(\mathrm{r}=0.19 ; \mathrm{p}<0.01)$, Pensamiento desiderativo $(\mathrm{r}=0.22 ; \mathrm{p}<0.01)$ y Retirada social ( $\mathrm{r}=0.17$; $\mathrm{p}<0.05)$, al mismo tiempo que una relación negativa con Expresión de emociones $(\mathrm{r}=-0.17 ; \mathrm{p}<0.05)$. Finalmente, el estilo de gestión conductual de conflictos de Integración se relaciona positivamente con las estrategias Resolución de problemas $(\mathrm{r}=0.29 ; \mathrm{p}<$ $0.01)$, Reestructuración cognitiva $(\mathrm{r}=0.22 ; \mathrm{p}<$ $0.05)$, Expresión de emociones $(r=0.18 ; \mathrm{p}<0.05)$, Apoyo social $(\mathrm{r}=0.19 ; \mathrm{p}<0.05)$ y Autocrítica $(\mathrm{r}=0.21 ; \mathrm{p}<0.05)$, al mismo tiempo que lo hace en sentido negativo con Retirada Social $(\mathrm{r}=$ $-0.17 ; \mathrm{p}<0.05)$.

TABLA 3

Matriz de correlaciones entre las escalas del ROCIII y SCI

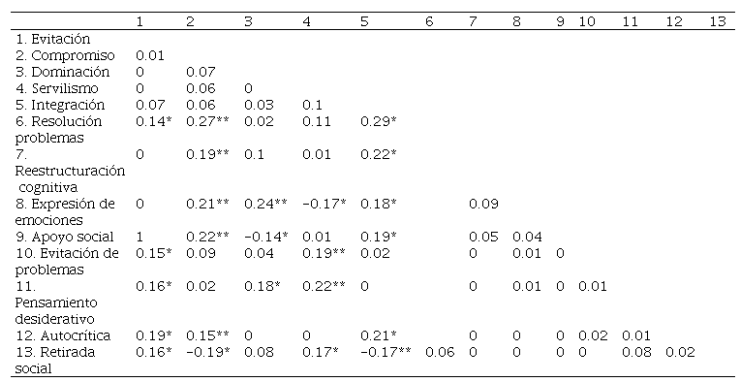

Nota*p $>0.05 * \mathrm{p}>0.001$

Fuente: elaboración propia.

\section{Discusión y Conclusiones}

Los resultados obtenidos en este estudio muestran que los orientadores laborales presentan unos niveles muy elevados en Ansiedad-Rasgo pero, sobre todo, en AnsiedadEstado, además de factores comunes a profesiones asistenciales como son la gran demanda de ayuda de los usuarios, atención a colectivos con graves dificultades socioeconómicas, excesiva burocratización del trabajo, y poco margen para tomar decisiones. En el caso de los orientadores laborales, hay que sumar la situación actual socieconómica/ nivel desempleo/expectativas. Actualmente la persona usuaria de servicios de orientación, en general se encuentra en una situación muy complicada, el tiempo de desempleo medio es mayor, el número de miembros desempleados en las familias aumenta, las prestaciones-subsidiosayudas se agotan. Por ello, el profesional desarrolla su trabajo con personas que llegan muy desmotivadas, con una importante y a veces dramática necesidad económica y sin demasiadas perspectivas de mejora. Precisamente esta última variable, la expectativa de empleo, es también fuente de estrés y generadora de ansiedad, tanto en usuarios como en orientadores; entre estos últimos la opinión generalizada es no saber qué alternativas y recursos de empleo ofrecer a las personas desempleadas en la actualidad, ya que las opciones de inserción laboral son muy escasas.

Con respecto a los estilos preferentemente utilizados por los orientadores para gestionar los conflictos en el contexto laboral, aparecen Evitación y Dominación, en contraposición a los estilos menos empleados, el Compromiso y la Integración. Esta circunstancia nos habla del uso frecuente de estilos más "tensionales" por esta muestra de profesionales para tratar de solucionar los problemas que les surgen en su trabajo; o los evitan y no los afrontan de manera directiva, o los abordan desde una postura desigual con respecto a la otra parte involucrada en el mismo. La Evitación, como una de los dos estrategias mayoritarias de gestión de conflicto aparecidas, podría explicarse como consecuencia de la ambigüedad de rol de los profesionales a la hora de buscar soluciones, asumiendo que no poseen responsabilidad para gestionar el conflicto, no les corresponde a ellos esa tarea, y creen que la institución para la que trabajan debería ser la responsable de dar soluciones. Por otro lado, también puede explicarse a través de la "difusión de responsabilidad", ya que el orientador forma parte de un equipo mucho más amplio y sobre todo pertenece a una estructura 
organizacional muy amplia (en el caso de los participantes en nuestro estudio, pertenecen al Servicio Andaluz de Empleo y coexisten con técnicos de oficinas de empleo, funcionarios de la Consejería de Empleo, del Servicio Público de Empleo estatal, gestores de empleo, etc.). En estos contextos tan difusos a la hora de trazar tareas específicas, suele ser habitual creer que la responsabilidad (en el momento de la aparición y/o solución de un conflicto), recae en otros y nunca en uno mismo. En cuanto al estilo de Dominación, como preferente también para la gestión de los conflictos, puede relacionarse con el "rol del experto" del orientador. En este caso, no se evita el conflicto, pero se afronta con la premisa de ser el que "sabe" qué ocurre y cómo solucionarlo.

Por otra parte, en cuanto a las relaciones obtenidas entre los estilos de gestión conductual del conflicto y la ansiedad, encontramos correlaciones positivas entre esta (tanto estado como rasgo) y los estilos de Evitación, Dominación y Servilismo, y negativamente con Integración y Compromiso. Resulta plausible pensar que a mayor nivel de ansiedad, menos complejo es el modo en el que el orientador va a gestionar el conflicto. De ahí que a mayor ansiedad mostrada, se tienda en mayor medida a evitar el conflicto, mostrarse sumiso a la otra parte o, por el contrario, a adoptar el papel dominador haciendo valer la propia postura unilateralmente. Por el contrario, la gestión del conflicto de manera integrativa o comprometida nos llevaría a la necesidad de una mayor estabilidad emocional, ya que para estos estilos de gestión es necesario poner en marcha competencias relacionadas con resolución de problemas complejos y procesos psicológicos superiores, que difícilmente se pueden activar con altos niveles de ansiedad.

Por otro lado, como ya se ha mencionado anteriormente, los conflictos no dejan de ser situaciones estresantes generadoras de un importante componente ansioso, de ahí que haya interesado establecer la incidencia de las principales estrategias de afrontamiento utilizadas por los orientadores, así como las posibles relaciones entre dichas estrategias de afrontamiento al estrés en general y los estilos de gestión conductual de los conflictos en particular. En primer lugar, se encuentran como estrategias más utilizadas por los orientadores el Pensamiento Desiderativo seguido de Evitación de problemas; estas estrategias reflejan un afrontamiento pasivo y desadaptativo centrado en el problema donde, $\mathrm{o}$ bien se evitan o niegan pensamientos o situaciones relacionadas con el hecho estresante, o se fantasea sobre realidades alternativas (a través de cogniciones que reflejan el deseo de que la realidad no fuera estresante).

En cuanto a las relaciones entre estilos de gestión de conflictos y estrategias de afrontamiento, se encuentra una correlación positiva entre el estilo de gestión de Evitación y las estrategias de afrontamiento relacionadas con la Evitación de problemas, Pensamiento desiderativo, Autocrítica y Retirada Social. Esto implica que aquellos orientadores que como forma general de afrontar situaciones estresantes llevan a cabo un manejo inadecuado de la situación a través de un afrontamiento pasivo y desadaptativo bien centrado en el problema (Pensamiento desiderativo y Evitación del problema), bien en la emoción (Autocrítica y Retirada Social), son quienes a la hora de abordar la situación estresante (el conflicto, en este caso), no lo hacen directamente; prefieren evitarlo, negarlo como modo de gestionarlo, en donde además pueden entrar en una dinámica de autoinculpación o autocrítica (por la ocurrencia de la situación estresante o su manejo inadecuado), cogniciones sobre realidades alternativas a la real o aislamiento social progresivo (al retirarse de aquellas personas que emocionalmente se encuentran asociadas al foco del conflicto).

Por otro lado, los estilos de gestión conductual del conflicto Compromiso e Integración, tienen los mismos patrones correlacionales, en el sentido de asociaciones positivas con las estrategias Resolución de problemas, Reestructuración cognitiva, Expresión de emociones, Apoyo social y Autocrítica, que implican relacionar, excepto en lo que se refiere a Autocrítica, una gestión del conflicto comprometido, complejo, que requiere estrategias de planificación, análisis y búsqueda de soluciones conjuntas, con el manejo 
adecuado de la situación estresante a través de un afrontamiento adaptativo, centrado en el problema (Resolución de problemas y Reestructuración cognitiva), o en la emoción (Expresión de emociones y Apoyo social). En este caso, quienes se comprometen con la gestión del conflicto son aquellos que activamente pueden poner en marcha estrategias cognitivas o conductuales para reducir el estrés (modificando la situación o el significado de la misma), al mismo tiempo que tienden al manejo adecuado de las emociones que aparecen durante la situación estresante y tienen un adecuado posicionamiento social (derivado de la correlación positiva con Apoyo Social y negativa con Retirada social) con su entorno. En relación a la asociación que ambos estilos de gestión conductual del conflicto mantienen con Autocrítica, la podríamos interpretar en el sentido que la autorreflexión sobre la responsabilidad (y no autoinculpación irracional) en la situación estresante (en su caso, el conflicto) puede ayudar a la búsqueda de un compromiso para su resolución.

De otro lado, el estilo de gestión de conflicto Dominación correlaciona positivamente con las estrategias de afrontamiento Expresión de emociones y Pensamiento desiderativo, al mismo tiempo que en sentido negativo con Apoyo Social. Empezando por esta última, puede que quien cree no tener apoyo social prefiera gestionar el conflicto (y por qué no, generalizarlo a otros estilos de respuesta interpersonales) desde la Dominación, o bien que quién opta por ser dominante en la gestión de conflictos tiende a ir perdiéndolos. Por otra parte, los orientadores que intentan gestionar verticalmente el conflicto desde una posición de superioridad con respecto a la otra parte, se pueden también caracterizar indistintamente por la puesta en marcha de estrategias adecuadas de manejo de la emoción (expresión y ventilación de emociones) como inadecuadas de manejo del pensamiento (cogniciones en el sentido de que la realidad no fuera estresante). Por último, en relación al Servilismo como estilo de gestión conductual del conflicto, se relaciona claramente con la utilización de estrategias de afrontamiento al estrés más desadaptativas, como Evitación de problemas, Pensamiento desiderativo y Retirada social.

En esta investigación se han evidenciado los importantes niveles de ansiedad que presenta el colectivo de profesionales de la orientación laboral, así como la vinculación que tienen a este componente tanto los estilos de gestión conductual de conflictos como las estrategias de afrontamiento al estrés, por lo que queda de manifiesto que ambos se encuentran conceptualmente muy próximos. Como principal limitación, resaltaríamos el tamaño de la muestra que, de haber sido más elevado hubiera permitido dividir a los participantes en grupos con diferentes niveles de ansiedad para determinar la relación con las estrategias de gestión de conflictos utilizadas.

\section{Agradecimientos}

Nuestro agradecimiento a todos los profesionales de la orientación laboral por su participación voluntaria en este trabajo.

\section{Referencias}

Basler, H. D., Kaluza, G., \& Lledó-Boyer, A. (2003). Evaluación de un programa de salud de afrontamiento al estrés. Ansiedad y Estrés, 9, 85-91.

Cano, F. J., Rodríguez, L., \& García, J. (2006). Adaptación española del Inventario de Estrategias de Afrontamiento. Actas Españolas de Psiquiatría, 35, 29-39.

Cuntigh, P., Perez, C., \& Personnaz, E. (2006). L 'Orientation Professionnelle des chômeurs en Europe. Bref, 232, 1-4. Recuperado de h ttp://www.cereq.fr/pdf/b232.pdf.

Di Fabio, A., \& Bernaud, J. L. (2008). The help-seeking in career counseling, Journal of Vocational Behavior, 72 (1), 60-66.

De Dreu, C. K. W., Van Dierendonck, D., \& Dijskstra, M. T. M. (2004). Conflict at work and individual well-being. International Journal of Conflict Management, 15, 6-26. 
De Pablo, J. M. (1995). Desarrollo de los aspectos personales para la ocupación: una metodología para el cambio con grupos de desempleados. Intervención Psicosocial, 5, 75-101.

Dewe, P. J. (1989). Examining the nature of work stress: individual evaluations of stressful experiences and coping. Human Relations, 42, 993-1013.

Secretaría de Estado de Administraciones Públicas (2015). Resolución de 26 de noviembre: Protocolo de actuación frente a la violencia en el trabajo en la Administración General del Estado y los organismos públicos vinculados o dependientes de ella. Madrid: BOE.

Fernández-Garrido, J. (2010). El tiempo y el espacio de las actividades de orientación. Cuadernos del Mercado de Trabajo, 5, 43-52.

Lázaro, S. (2004). El desgaste profesional en los trabajadores sociales. Portularia, 4, 499-506.

Lazarus, R. S. (2000). Estrés y emoción. Manejo e implicaciones en nuestra salud. Bilbao: Desclée Brouwer.

Maslach, C., Schaufeli, W., \& Leiter, M. (2001). Job Burnout. Annual Review of Psychology, 52 (1), 397-422.

Munduate, L., Ganaza, J., \& Alcaide, M. (1993). Estilos de gestión del conflicto interpersonal en las organizaciones. Revista de Psicología Social, 8, 47-68.

Munduate, L. \& Medina, F. J. (2006). Gestión del conflicto, negociación y mediación. Madrid: Pirámide.

Organización Internacional del Trabajo (2003). Repertorio de recomendaciones prácticas sobre la violencia en el lugar de trabajo en el sector de los servicios y medidas para combatirla. Recuperado de http://www.ilo.org/public/spanish/stand ards/relm/gb/docs/gb288/pdf/mevsws-11.pd f/

Pole Emploi (2009). Notre enquete sur la malaise des conseillers d'emploi. Recuperado de http://lentreprise.lexpress.fr/rh-manage ment/formation/pole-emploi-notre-enquet e-sur-le-malaise-des-conseillers-en-placem ent_1323515.html.
Rahim, M.A. (1983): A measure of styles of handling interpersonal conflict. Academy of Management Journal, 26(2), 368-376.

Schaufeli, W. B. (1998). The burnout companion to study and practice: A critical analysis. London: Taylor \& Francis.

Seisdedos, N. (1988). Cuestionario de ansiedad estado-rasgo. Manual. Madrid: TEA.

Spector, P. E., Chen, P. Y., \& O'Connell, B. J. (2000). A longitudinal study of relations between job stressors and job strains while controlling for prior negative affectivity and strains. Journal of Applied Psychology, 85, 211-218.

Spielberger, C.D., Gorsuch, R.L., \& Lushene, R. E. (1970). Manual for the State-Trait Anxiety Inventory. Palo Alto: Consulting Psychologist Press.

Tobin D. L., Holroyd K. A., Reynolds R. V., \& Kigal J. K. (1989). The hierarchical factor structure of the Coping Strategies Inventory. Cognitive Therapy and Research, 13-343-61.

Van Woerkom, M., \& Van Engen, M. L. (2009). Learning from conflicts? The relations between task and relationship conflicts, team learning and team performance. European Journal of Work and Organizational Psychology, 18(4), 381-404.

\section{Notas}

* Artículo de Investigación. 